\title{
Screening, Coulomb pseudopotential, and superconductivity in alkali-doped Fullerenes
}

\author{
Erik $\operatorname{Koch}^{(a, b)}$, Olle Gunnarsson ${ }^{(a)}$, and Richard M. Martin ${ }^{(b)}$ \\ ${ }^{(a)}$ Max-Planck-Institut für Festkörperforschung, 70506 Stuttgart, Germany \\ ${ }^{(b)}$ Department of Physics, University of Illinois at Urbana-Champaign, Urbana, IL 61801, USA
}

(August 8, 2021)

\begin{abstract}
We study the static screening in a Hubbard-like model using quantum Monte Carlo. We find that the random phase approximation is surprisingly accurate almost up to the Mott transition. We argue that in alkali-doped Fullerenes the Coulomb pseudopotential $\mu^{*}$ is not very much reduced by retardation effects. Therefore efficient screening is important in reducing $\mu^{*}$ sufficiently to allow for an electron-phonon driven superconductivity. In this way the Fullerides differ from the conventional picture, where retardation effects play a major role in reducing the electron-electron repulsion.
\end{abstract}

74.70.Wz,71.10.Fd,71.20.Tx

The random phase approximation (RPA) has been very widely used in solid state physics. It properly describes the screening when the kinetic energy is much larger than the interaction energy. In the opposite limit, however, the RPA is qualitatively wrong. Little is known about the more interesting situation when the two energies are comparable. In this paper we show for a Hubbard-like model that the RPA gives a surprisingly accurate description of the static screening on the metallic side of a Mott transition until the system is close to the transition.

For conventional superconductors the electron-phonon interaction leads to an effective electron-electron attraction. This interaction is counteracted by the strong Coulomb repulsion, which is, however believed to be strongly reduced by retardation effects. 2 . 3 The resulting effective Coulomb interaction is described by the dimensionless Coulomb pseudopotential $\mu^{*}$, which is believed to be typically of the order 0.1 . Here we argue that the situation for $\mathrm{A}_{3} \mathrm{C}_{60}(\mathrm{~A}=\mathrm{K}, \mathrm{Rb})$ is different. We find that retardation effects are rather inefficient. Therefore the screening of the Coulomb interaction becomes important for reducing the electron-electron repulsion. Thus, although the superconductivity in $\mathrm{A}_{3} \mathrm{C}_{60}$ is driven by the electron-phonon interaction, $\theta$ the origin of the strong reduction of $\mu^{*}$ is different from the current picture of conventional superconductors. In the scenario we are putting forward, several puzzling phenomena find a natural explanation. In $\mathrm{A}_{3} \mathrm{C}_{60}(\mathrm{~A}=\mathrm{K}, \mathrm{Rb})$ the transition temperature $T_{c}$ is reduced by pressure $\mathrm{O}_{\text {For }} \mathrm{Cs}_{3} \mathrm{C}_{60}$, however, which only under pressure becomes a superconductor, $T_{c}$ increases with pressure. This is consistent with the picture where $\mu^{*}$ is reduced by screening, since the screening is less efficient close to a Mott transition. Second, it was very early pointed out that the alkali phonons ought to couple efficiently to the electrons 0 although later experiments showed that this was not the case. We show that efficient screening reduces the coupling to the alkali phonons.

We first discuss the screening in the RPA. In the random phase approximation it only costs kinetic energy to screen a test charge. In the limit where a typical Coulomb integral $U$ is large compared with the band width $W$, the kinetic energy cost of screening is relatively small compared with the potential energy gain, so the screening is efficient. This means that as a test charge $q$ is introduced on a site $c$, almost the same amount of electronic charge moves away from the site, leaving it almost neutral. This argument neglects, however, that when an electron leaves a site it has to find another site with a missing electron or there is a large Coulomb energy penalty. Thus the RPA is accurate for small values of $U / W$, while it is qualitatively wrong for large values. It is not clear what happens for intermediate values.

To study the screening in $\mathrm{A}_{3} \mathrm{C}_{60}$, we use a Hubbard-like model, including the three-fold degenerate $t_{1 u}$ orbital:

$$
\begin{aligned}
H & =\sum_{<i j>} \sum_{m m^{\prime} \sigma} t_{i m, j m^{\prime}} \psi_{i m \sigma}^{\dagger} \psi_{j m^{\prime} \sigma} \\
& +U \sum_{i} \sum_{(m \sigma)<\left(m^{\prime} \sigma^{\prime}\right)} n_{i m \sigma} n_{i m^{\prime} \sigma^{\prime}}+q U \sum_{m \sigma} n_{c m \sigma} .
\end{aligned}
$$

The first term describes the kinetic energy, the second term the on-site Coulomb interaction and the third term the interaction with the test charge $q$ on site $c . \psi_{i m, \sigma}$ annihilates an electron on site $i$ with orbital quantum number $m$ and spin $\sigma$, and $n_{i m \sigma}=\psi_{i m \sigma}^{\dagger} \psi_{i m \sigma}$. The effect of orientational disorder 10 is built into the hopping integrals $t_{i m, j m^{\prime}}$. The band width is about $0.63 \mathrm{eV}$. Multiplet effects are not included, but we remark that they tend to be counteracted by the Jahn-Teller effect which is also neglected. The test charge is assumed to interact with the electrons on the same site via the Coulomb integral $U$. The system has three electrons per molecule, i.e. a half-filled $t_{1 u}$ band.

We have investigated the model by using a lattice diffusion quantum Monte Carlo (QMC) method.11.12 In this method a trial function $\left|\psi_{T}\right\rangle$ is constructed and allowed to diffuse towards the exact solution, under the constraint of a fixed node approximation $\left|\psi_{T}\right\rangle$ is obtained from a generalized Gutzwiller Ansatz 4

$$
\left|\Psi_{T}\right\rangle=g^{D} g_{0}^{n_{c}}\left|\Psi_{0}\right\rangle
$$


where $\left|\Psi_{0}\right\rangle$ is a Slater determinant constructed from solutions of eqn. (11) in the Hartree approximation, $D$ is the number of double occupancies in the system, and $n_{c}$ is the number of electrons on site $c . g$ and $g_{0}$ are variational parameters. $g^{D}$ is the usual Gutzwiller factor while $g_{0}^{n_{c}}$ allows us to optimize the charge on the site with the test charge. In addition to the DMC calculation, we also perform a variational Monte Carlo calculation (VMC) and the energy is minimized as a function of $g$ and $g_{0} .13$ In all cases the state is assumed to be paramagnetic. For $U / W \sim 2.5$ there is a transition to an antiferromagnetic Mott insulator 12 where the screening is very inefficient. Here, however, we focus on $U / W<2.5$. We obtain the charge on site $c$ from the extrapolated estimator $n_{c} \approx 2 n_{c}(D M C)-n_{c}(V M C)$, where $n_{c}(V M C)$ is the expectation value for the wave function (2) calculated by $\mathrm{VMC}$ and $n_{c}(D M C)$ is the mixed estimator from the DMC calculation.

To test the accuracy of the approach, which involves the fixed-node approximation and uses the extrapolated estimator, we have compared the results of our QMC calculations with the the exact results from exact diagonalization of a system with four molecules (12 electrons). The comparison shown in Fig. 1 illustrates that the QMC calculations are quite accurate for the system we are analyzing here.

Since we are interested in the linear response, we should calculate the effect of an infinitesimally small test charge $q$. Because of the statistical error in a QMC calculation it is, however, difficult to determine the response to a small perturbation. To get a good signal-to-noise ratio, we would therefore like to use as large a test charge as possible. To estimate how large we can make $q$ and still be in the linear response regime, we have performed Lanczos calculations for a range of different test charges. We find that for $q \leq 0.25 e$ the response is practically linear.

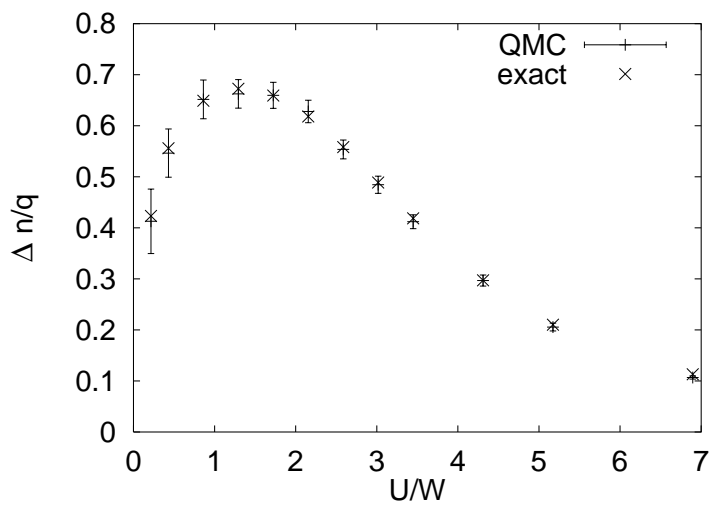

FIG. 1. Screening charge $\Delta n$ on the site of the test charge $(q=0.25 e)$ as a function of $U / W$, where $U$ is the Coulomb interaction and $W$ is the band width. Exact diagonalization and QMC calculations have been performed for four molecules (12 electrons). The figure shows that the QMC calculations are quite accurate over the whole range of $U / W$.

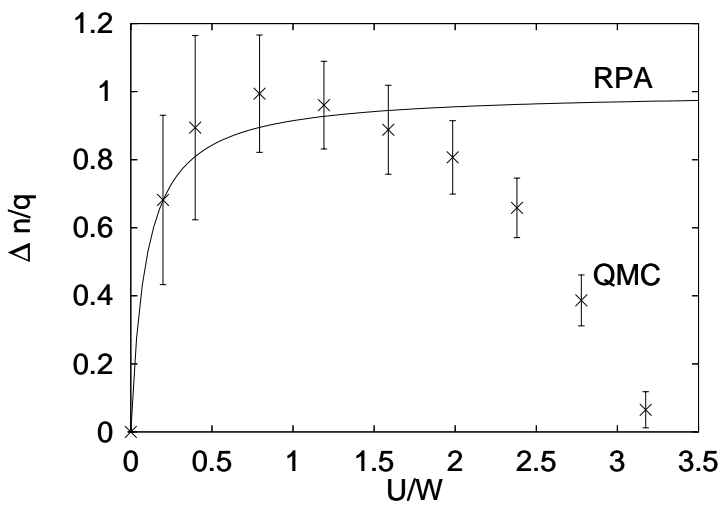

FIG. 2. Screening charge $\Delta n$ on the site of the test charge $(q=0.25 e)$ as a function of $U / W$, extrapolated to infinite cluster size. The full curve shows the screening charge in the RPA, obtained from Hartree calculations for the Hamiltonian (1). The crosses with errorbars give the results of the QMC calculations. The RPA screening remains rather accurate up to $U / W \sim 2$, but fails badly for larger values of $U / W$. The screening is very efficient for $U / W \sim 0.5-2.0$.

We have performed QMC calculations for larger clusters of $N_{\mathrm{mol}}=32,48,64,72$, and 108 molecules, where exact diagonalization is not possible. The screening charge $\Delta n_{c}=n_{c}(0)-n_{c}(q)$ was extrapolated to infinite cluster size, assuming a finite-size scaling of the form $\Delta n_{c}\left(N_{\mathrm{mol}}\right)=\Delta n_{c}+\alpha / N_{\mathrm{mol}}$. The results are shown in Fig. 2. For rather small values of $U / W(\sim 0.5-1.0)$, the RPA somewhat underestimates the screening. Such a behavior is also found in the electron gas.15 For intermediate values of $U / W(\sim 1.0-2.0)$ the RPA gives surprisingly accurate results. This is one of the main results of this paper. For large $U / W$, the RPA rapidly becomes qualitatively wrong, as discussed earlier. We are now in the position of addressing the superconductivity in $\mathrm{A}_{3} \mathrm{C}_{60}$.

In the theory of superconductivity, a dimensionless quantity $\mu^{*}$, the Coulomb pseudopotential, is introduced to describe the effects of the Coulomb repulsion. One introduces $\mu=U N(0)$, where $U$ is a typical screened Coulomb interaction and $N(0)$ is the density of states per spin at the Fermi energy. Retardation effects renormalize $\mu$ to $\mu^{*}$, and are described by ladder diagrams in the statically screened Coulomb interaction. 2.3 It is found that

$$
\mu^{*}=\frac{\mu}{1+\mu \ln \left(\omega_{e l} / \omega_{p h}\right)} \sim \frac{1}{\ln \left(\omega_{e l} / \omega_{p h}\right)}
$$

where $\omega_{e l}$ and $\omega_{p h}$ are typical electron and phonon energy scales, respectively. Often $\mu \ln \left(\omega_{e l} / \omega_{p h}\right)$ is substantially larger than unity. In that limit the last part of eqn. (3) holds, i.e. $\mu^{*}$ is determined solely by retardation effects, independently of the screening, which only changes $\mu$.

In solid $\mathrm{C}_{60}$ we have many narrow subbands $(\sim 0.5 \mathrm{eV}$ 
wide), spread over a range of about $30 \mathrm{eV}$. In the traditional approach one assumes that the relevant energy range extends over all this region. Summing the ladder diagrams in the screened Coulomb interaction leads to a large renormalization of $\mu$. Exact results for a two-band model show, however, that in the appropriate limits this approach greatly overestimates the renormalization due to the upper sub band 16 In the limit when a sub band is far away from the Fermi energy, the correct approach is to first project out the high energy degrees of freedom corresponding to this sub band. This leads to an effective Hamiltonian, expressed in terms of the unscreened Coulomb matrix elements, which describes the low energy properties of the system. The main difference between the two approaches is the order in which high and low energy degrees of freedom are treated. In the traditional approach the Coulomb interaction is screened first, which in particular involves the low energy degrees of freedom. After this the high energy degrees of freedom are projected out. This approach involves uncontrolled approximations. Our approach, instead, projects out the high energy degrees of freedom first, and it allows us to make statements about the importance of these degrees of freedom. Although these arguments were presented in the context of $\mathrm{C}_{60}$, they are rather general. We now make more specific arguments for $\mathrm{C}_{60}$ to provide further evidence that the retardation effects from higher sub bands are not very large.

From Auger measurements on $\mathrm{K}_{6} \mathrm{C}_{60}$, the Coulomb interaction $U$ between two holes in an otherwise full $t_{1 u}$ band has been estimated to about $1.5 \mathrm{eV} 10$ This reduction of $U$ from about $4 \mathrm{eV}^{18}$ for a free molecule to about $U_{\text {insul }}=1.5 \mathrm{eV}$ for the the insulating solid, is mainly due to intramolecular processes and to polarization of the molecules surrounding the two holes. Since the excitation energy of the relevant final state in the Auger experiment is rather small (about $1.5 \mathrm{eV}$ ), $U_{\text {insul }}$ should contain the renormalization from all the higher sub bands, except possibly the ones closest to the $t_{1 u}$ band. If we multiply $U_{\text {insul }}$ by $N(0) \sim 6,19$ the result is a very large $\mu \sim 9$, much too large to allow for a phonon induced superconductivity unless $\mu$ is further reduced by other effects.

In $\mathrm{K}_{3} \mathrm{C}_{60}$ screening and retardation effects inside the $t_{1 u}$ band become available. The argument against summing ladder diagrams in the screened interaction were only justified for higher sub bands. Within the $t_{1 u}$ band we therefore rely on this conventional theory, 2,3 which in addition usually uses Thomas-Fermi or RPA screening. A priori, the use of RPA seems highly questionable for these strongly correlated systems. Our calculations, however, support this approximatiom unless the system is close to a Mott transition. Taking the long range Coulomb interaction into account, the RPA screening reduces $\mu$ to about 0.4.16 Including the additional retardation effects inside the $t_{1 u}$ band according to eqn. (3) finally renormalizes $\mu$ to $\mu^{*} \approx 0.3$. Thus the Coulomb pseudopotential is primarily reduced by screening and not by retardation effects. In contrast, using eqn. (3) with $\omega_{e l} \approx 15 \mathrm{eV}$ and $\omega_{p h} \approx 0.1 \mathrm{eV}$ would result in $\mu^{*} \lesssim 0.2$, practically independent of $\mu$. A Coulomb pseudopotential $\mu^{*} \approx 0.3$ is substantially larger than for conventional superconductors, $\mathrm{l}$ but it is not so large that it prevents the superconductivity from being driven by the electronphonon interaction. 20 Recent tunneling experiments give $\mu^{*}=0.329$ for $\mathrm{Rb}_{3} \mathrm{C}_{60} .21$

We now turn to the question how $T_{c}$ changes with the lattice constant $a$. The main effect of increasing $a$ is to decrease the band-width $W$ and increase the density of states at the Fermi level $N(0)$. Using McMillan's formula, $T_{c}$ is given by

$$
T_{c}=\frac{\omega_{p h}}{1.2} \exp \left[\frac{-1.04(1+\lambda)}{\lambda-\mu^{*}(1+0.62 \lambda)}\right],
$$

with $\lambda=N(0) V$ the electron-phonon coupling constant. $\mu^{*}$ is calculated from $\mu=N(0) U_{\text {insul }}(1-\gamma)$, where $U_{\text {insul }}$ is a typical unscreened Coulomb matrix element and $\gamma=d n / d q$ describes the screening within the $t_{1 u}$ band. Assuming that $\omega_{e l}$ in Eq. (3) is large, $\mu^{*}$ is practically independent of the lattice constant $a$. Since $N(0)$ increases with decreasing $a$, the electron-phonon coupling $\lambda$ becomes stronger, increasing $T_{c} 22$ Assuming a small $\omega_{e l}$, corresponding to the $t_{1 u}$ band width, it is no longer true that $\mu^{*}$ is independent of $\mu$. However, if the RPA is valid, $\mu$ is almost independent of the lattice constant, since the increase in $N(0)$ is counteracted by a slightly more efficient screening $\gamma_{R P A}$ (cf. Fig. 2). Hence also in this scenario we find that $T_{c}$ increases with $a$. But what happens when the lattice constant $a$ becomes large enough that we enter the region where the screening starts to break down? Then $\mu$ will start to increase considerably with $a$. Assuming a large $\omega_{e l}, \mu^{*}$ is still independent of $\mu$, and therefore $T_{c}$ should keep increasing. For small $\omega_{e l}$, on the other hand, $\mu^{*}$ will start to rapidly increase with $a$, leading to a steep drop in $T_{c}$. This resembles the anomalous behavior observed in $\mathrm{Cs}_{3} \mathrm{C}_{60}$ : it only becomes superconducting under pressure, with $T_{c}$ rapidly decreasing with increasing lattice constant.6

It might appear that efficient screening is not really helpful for superconductivity. Phonons couple to the electrons by perturbing the potential seen by the electrons. 3 An example being the longitudinal modes of a jellium. Efficient screening tends to weaken the coupling to such phonons, since it reduces the perturbation considerably. To some extent, such a reduction also seems to be at work in $\mathrm{C}_{60}$. Initially it was expected that the coupling to the alkali phonons would be very strong. 0 Each $\mathrm{C}_{60}$ molecule is surrounded by 14 alkali ions with relatively weak force constants. When an electron arrives on a $\mathrm{C}_{60}$ molecule one would therefore expect that the surrounding alkali ions respond strongly. This was, however, not confirmed by experiment. For instance, an alkali isotope effect could not be observed within the experimental accuracy. 8 This finding can be naturally understood as an effect of the efficient screening found in our calculations. When an electron arrives 
on a $\mathrm{C}_{60}$ molecule, other electrons leave the molecule, which thus stays almost neutral. The alkali ions then only see a small change in the net charge and therefore couple weakly. In a similar way it follows that intramolecular phonons of $\mathrm{A}_{g}$ symmetry couple weakly. An $\mathrm{A}_{g}$ phonon shifts all the $t_{1 u}$ levels on a given molecule in the same direction. This shift of the center of gravity can be screened very efficiently by transferring charge from the molecules where the levels move upwards to those where they move downwards. The modes that are important for the superconductivity in solid $\mathrm{C}_{60}$ are, however, different. An intramolecular $\mathrm{H}_{g}$ phonon does not shift the center of gravity of the $t_{1 u}$ level. Thus the $\mathrm{H}_{g}$ phonons are not screened by the transfer of charge. Hence for these phonons the efficient screening serves to reduce $\mu^{*}$ without affecting the electron-phonon coupling.

To summarize, we have calculated the static screening of a point charge for a Hubbard-like model using quantum Monte Carlo. We find that the RPA is surprisingly accurate up to values of $U / W$ fairly close to the Mott transition. For larger $U / W$ the screening rapidly breaks down. This result should have quite general implications for the physics of systems close to a Mott transition. Here we have studied the consequences for the superconductivity in the alkali-doped Fullerenes. We have provided arguments that for $\mathrm{A}_{3} \mathrm{C}_{60}(\mathrm{~A}=\mathrm{K}, \mathrm{Rb})$ retardation effects are very inefficient in reducing the electron-electron repulsion. Instead, and unlike for textbook superconductors, screening is mainly responsible for the reduction of the Coulomb pseudopotential $\mu^{*}$. This results in a $\mu^{*}$ small enough that the electron-phonon interaction can drive the superconductivity. Nevertheless $\mu^{*}$ is substantially larger than for conventional superconductors, in agreement with recent experiments. This scenario is quite different from the conventional picture of a superconductor, where the retardation effects are believed to play the central role in reducing $\mu^{*}$. It explains quite naturally the anomalous pressure dependence of $T_{c}$ found for $\mathrm{Cs}_{3} \mathrm{C}_{60}$ and the absence of a strong coupling to the alkali phonons. It also predicts that the coupling to the $\mathrm{A}_{g}$ phonons is strongly reduced by screening effects. Finally, our results let us understand the surprising fact that $T_{c}$ peaks for systems close to the Mott transition, where the density of states is large, but the screening has not yet started to become inefficient.

This work has been supported by the Alexander-vonHumboldt-Stiftung under the Feodor-Lynen-Program and the Max-Planck-Forschungspreis, and by the Department of Energy, grant DEFG 02-96ER45439.

\footnotetext{
${ }^{1}$ D. Pines, Elementary excitations in solids, Benjamin (New York, 1964).
}

2 N.N. Bogoliubov, Nuovo Cimento, 7, 6, 794 (1958); P. Morel, and P.W. Anderson, Phys. Rev. 125, 1263 (1962).

${ }^{3}$ J.R. Schrieffer, Theory of superconductivity, Benjamin, (New York, 1964); D.J. Scalapino, in Superconductivity, Ed. R.D. Park, Dekker (New York, 1969), pp. 449; V.L. Ginzburg and D.A. Kirzhnits, High-Temperature Superconductivity, Consultants Bureau (New York, 1982).

${ }^{4}$ O. Gunnarsson, Rev. Mod. Phys. 69, 575 (1997).

${ }^{5}$ R.M. Fleming, A.P. Ramirez, M.J. Rosseinsky, D.W. Murphy, R.C. Haddon, S.M. Zahurak, and A.V. Makhija, Nature 352, 787 (1991).

${ }^{6}$ T.T.M. Palstra, O. Zhou, Y. Iwasa, P.E. Sulewski, R.M. Fleming, and B.R. Zegarski, Solid State Commun. 93, 327 (1995).

${ }^{7}$ F.C. Zhang, M. Ogata, and T.M. Rice, Phys. Rev. Lett. 67, 3452 (1991).

${ }^{8}$ T.W. Ebbesen, J.S. Tsai, K. Tanigaki, H. Hiura, Y. Shimakawa, Y. Kubo, I. Hirosawa, and J. Mizuki, Physica C 203, 163 (1992); B. Burk, V.H. Crespi, A. Zettl, and M.L. Cohen, Phys Rev. Lett. 72, 3706 (1994).

${ }^{9}$ O. Gunnarsson, S. Satpathy, O. Jepsen, and O.K. Andersen, Phys. Rev. Lett. 67, 3002 (1991).

${ }^{10}$ I.I. Mazin, S.N. Rashkeev, V.P. Antropov, O. Jepsen, A.I. Liechtenstein, and O.K. Andersen, Phys. Rev. B 45, 5114 (1992).

${ }^{11}$ D.F.B. ten Haaf, H.J.M. van Bemmel, J.M.J. van Leeuwen, W. van Saarloos, and D.M. Ceperley, Phys. Rev. B 51, 13039 (1995); H.J.M. van Bemmel, D.F.B. ten Haaf, W. van Saarloos, J.M.J. van Leeuwen, and G. An, Phys. Rev. Lett. 72, 2442 (1994).

12 O. Gunnarsson, E. Koch and R.M. Martin, Phys. Rev. B 54, R11026 (1996); M. Calandra and S. Sorella, Phys. Rev. B 57, 11446 (1998); A.C. Cosentini, M. Capone, L. Guidoni, and G.B. Bachelet, Phys. Rev. B 58, R14685 (1998).

13 E. Koch, O. Gunnarsson, and R.M. Martin, preprint.

${ }^{14}$ M.C. Gutzwiller, Phys. Rev. 137, A1726 (1965).

${ }^{15}$ L. Hedin and S. Lundqvist, Solid State Physics Vol. 23, (Eds. H. Ehrenreich, D. Turnbull and F. Seitz, Academic, New York, 1969) pp. 1; S. Moroni, D.M. Ceperley, G. Senatore, Phys. Rev. Lett. 75, 689 (1995).

${ }^{16}$ O. Gunnarsson and G. Zwicknagl, Phys. Rev. Lett. 69, 957 (1992); O. Gunnarsson, D. Rainer, and G. Zwicknagl, Int. J. Mod. Phys. B 6, 3993 (1992).

${ }^{17}$ R.W. Lof, M.A. van Veenendaal, B. Koopmans, H.T. Jonkman, and G.A. Sawatzky, Phys. Rev. Lett. 68, 3924 (1992); P.A. Brühwiler, A.J. Maxwell, A. Nilsson, N. Mårtensson, and O. Gunnarsson, Phys. Rev. B 48, 18296 (1992).

18 V.P. Antropov, O. Gunnarsson, and O. Jepsen, Phys. Rev. B 46, 13647 (1992).

${ }^{19}$ F. Aryasetiawan, O. Gunnarsson, E. Koch, and R.M. Martin, Phys. Rev. B 55, R10165 (1997).

${ }^{20}$ O. Gunnarsson, H. Handschuh, P.S. Bechthold, B. Kessler, G. Ganteför, and W. Eberhardt, Phys. Rev. Lett. 74, 1875 (1995).

21 J.R. Ostrick, L.M. Merchant, F. Hellman, and R.C. Dynes, Bull. Am. Phys. Soc. 43, 27 (1998) and priv. comm.

${ }^{22}$ M. Schlüter, M. Lannoo, M. Needels, G.A. Baraff, and D. Tománek, J. Phys. Chem. Solids 53, 1473 (1992). 